\title{
Molecular frame photoelectron angular distribution for oxygen 1s photoemission from $\mathrm{CO}_{2}$ molecules
}

\author{
N Saito ${ }^{1}$, K Ueda ${ }^{2}$, A De Fanis ${ }^{2,8}$, K Kubozuka ${ }^{3}$, M Machida ${ }^{3}$, I Koyano ${ }^{3}$, \\ R Dörner ${ }^{4}$, A Czasch ${ }^{4}$, L Schmidt ${ }^{4}$, A Cassimi ${ }^{5}$, K Wang $^{6}$, \\ B Zimmermann ${ }^{6,7}$ and V McKoy ${ }^{6}$ \\ ${ }^{1}$ National Metrology Institute of Japan, National Institute of Advanced Industrial Science and \\ Technology (AIST), Tsukuba 305-8568, Japan \\ 2 Institute of Multidisciplinary Research for Advanced Materials, Tohoku University, \\ Sendai 980-8577, Japan \\ ${ }^{3}$ Department of Material Science, Himeji Institute of Technology, Kamigori, Hyogo 678-1297, \\ Japan \\ ${ }^{4}$ Institut für Kernphysik, Universität Frankfurt, D-60438 Frankfurt, Germany \\ 5 CIRIL/CEA/CNRS/ISMRA, Université de Caen, Box 5133, F-14070 Caen Cedex 5, France \\ ${ }^{6}$ A A Noyes Laboratory of Chemical Physics, California Institute of Technology, Pasadena, \\ CA 91125, USA \\ ${ }^{7}$ Max Planck Institute for the Physics of Complex Systems, 01187 Dresden, Germany \\ E-mail: norio.saito@aist.go.jp and ueda@tagen.tohoku.ac.jp
}

Received 22 April 2005, in final form 30 June 2005

Published 16 August 2005

Online at stacks.iop.org/JPhysB/38/L277

\begin{abstract}
We have measured photoelectron angular distributions in the molecular frame (MF-PADs) for $\mathrm{O} 1 \mathrm{~s}$ photoemission from $\mathrm{CO}_{2}$, using photoelectron- $\mathrm{O}^{+}-\mathrm{CO}^{+}$ coincidence momentum imaging. Results for the molecular axis at 0,45 and $90^{\circ}$ to the electric vector of the light are reported. The major features of the MF-PADs are fairly well reproduced by calculations employing a relaxed-core Hartree-Fock approach. Weak asymmetric features are seen through a plane perpendicular to the molecular axis and attributed to symmetry lowering by anti-symmetric stretching motion.
\end{abstract}

(Some figures in this article are in colour only in the electronic version)

Photoelectron angular distributions in the molecular frame (MF-PADs) have been investigated extensively for K-shell photoemission from the diatomic molecules $\mathrm{CO}$ and $\mathrm{N}_{2}$ [1-9]. The MF-PADs for molecules aligned parallel to the electric vector of the linearly polarized light exhibit rich structure in regions of shape resonances and provide a dynamical window on the underlying partial-wave composition of the photoelectron as it scatters off the anisotropic potential of the ion. It is worth noting that the observed MF-PADs for $\mathrm{N}_{2}$ display reflection

8 Present address: Shimadzu Research Laboratory, Wharfside, Trafford Wharf Road, Manchester, M17 1GP, UK. 
symmetry through a plane perpendicular to the molecular axis $[1,4,6,7]$, even if the photoemission is related to asymmetric $\mathrm{N}^{+}-\mathrm{N}^{++}$fragmentation [6]. On the other hand, those for $\mathrm{CO}$ are asymmetric [2, 3, 5, 8], due to the asymmetric nature of the hetero-nuclear diatom. Calculations employing Hartree-Fock and random-phase-approximation methods and an extension of the standard multiple scattering approach reproduce key features of the measured MF-PADs well [4, 7, 10].

MF-PADs for core-level photoemission from linear triatomic molecules have also been investigated. MF-PADs for $\mathrm{C} 1 \mathrm{~s}$ photoemission from $\mathrm{CO}_{2}[11,12]$ display reflection symmetry through a plane perpendicular to the molecular axis, even if the photoemission is related to the asymmetric $\mathrm{O}^{+}-\mathrm{CO}^{+}$fragmentation [12], while MF-PADs for $\mathrm{O} 1 \mathrm{~s}, \mathrm{C} 1 \mathrm{~s}$ and $\mathrm{S} 2 \mathrm{p}$ photoemission from OCS [13] are asymmetric, in analogy to $\mathrm{N}_{2}$ and $\mathrm{CO}$, respectively. Calculations employing a relaxed-core Hartree-Fock approach [12] and an extension of the standard multiple scattering approach [13] reproduce the measured MF-PADs reasonably well, but the agreement is less satisfactory than for the diatomic cases.

In the present work, we have extended our previous experimental and theoretical work on $\mathrm{C} 1 \mathrm{~s}$ MF-PADs [12] of $\mathrm{CO}_{2}$ to $\mathrm{O} 1 \mathrm{~s}$ MF-PADs. The $\mathrm{O} 1 \mathrm{~s}$ orbitals consist of symmetry-adapted molecular orbitals $1 \sigma_{g}$ and $1 \sigma_{u}$. The $\mathrm{O} 1 \mathrm{~s}$ ionization threshold is at $541.254 \mathrm{eV}$ [14]. On the $\mathrm{O} 1 \mathrm{~s}$ absorption spectrum, two shape resonances, $1 \sigma_{u} \rightarrow 5 \sigma_{g}^{*}$ and $1 \sigma_{g} \rightarrow 4 \sigma_{u}^{*}$, appear at $\sim 542 \mathrm{eV}$, very close to the $\mathrm{O} 1 \mathrm{~s}$ ionization threshold, and at $\sim 559 \mathrm{eV}$, about $18 \mathrm{eV}$ above the $\mathrm{O} 1 \mathrm{~s}$ ionization threshold, respectively [15]. The MF-PADs of $\mathrm{O} 1 \mathrm{~s}$ photoemission from $\mathrm{CO}_{2}$ have been previously reported only for the molecular axis parallel to the electric vector of the light [16]; ions, however, defining the molecular axis were not specified. In this letter, we report measured and calculated MF-PADs for $\mathrm{O} 1 \mathrm{~s}$ photoemission from $\mathrm{CO}_{2}$ across the shape resonances. In the experiment, we employ coincidence momentum imaging spectroscopy in which coincidence signals between the $\mathrm{O} 1 \mathrm{~s}$ photoelectron and the ion pair $\mathrm{O}^{+}-\mathrm{CO}^{+}$are selected [12]. In addition to the detailed comparison between the measured and calculated MF-PADs, it is of interest to see whether reflection asymmetry appears in the MF-PADs because of symmetry breaking induced by antisymmetric stretching vibrations in the $\mathrm{O} 1 \mathrm{~s}$ ionized state $[17,18]$.

The measurements were carried out on the c-branch of the figure- 8 undulator beam line $27 \mathrm{SU}$ at SPring-8, the $8 \mathrm{GeV}$ synchrotron radiation facility in Japan [19-21]. With acceptance angles employed in the present experiments, the degree of light polarization is almost $100 \%$ on the beam axis [22]. The storage ring was operated in the several singlebunches mode such as the 10 single-bunches $+1 / 12$ and 18 single-bunches $+1 / 21$ filling modes, which provide bunch intervals of 399.2 and $228.1 \mathrm{~ns}$, respectively, and permit electron TOF measurements.

Coincidence measurements were recorded at seven photon energies between 543 and $574 \mathrm{eV}$. Our coincidence momentum imaging apparatus is based on a measurement of electron and ion time of flight (TOF) with two multi-hit two-dimensional position sensitive detectors [23-25]. Similar apparatus are used by other groups as well [9, 26, 27]. A supersonic jet of $\mathrm{CO}_{2}$ in the vertical direction crosses the photon beam in the horizontal direction. The TOF axis is in the horizontal direction and perpendicular to both the gas and photon beams. Ions are accelerated by a uniform electrostatic field $\left(0.59 \mathrm{~V} \mathrm{~mm}^{-1}\right.$ for the measurement at the lowest photon energy $543 \mathrm{eV}$ and $1.12 \mathrm{~V} \mathrm{~mm}^{-1}$ for the measurements at other photon energies) to a micro-channel-plate (MCP) detector at one end of the acceleration region while the same field accelerates electrons to the other side where they enter the drift region. The lengths of the electron acceleration and drift regions are 70 and $140 \mathrm{~mm}$, respectively. A uniform magnetic field (2.4 G for $543 \mathrm{eV}$ and $10.2 \mathrm{G}$ for the other photon energies) helps drive the electrons towards the MCP detector at the end of the drift region [28]. Each MCP detector is 
fitted to a two-dimensional (2D) multi-hit readout delay-line anode (Roentdek ${ }^{9}$ ). This permits measurements of both the time of detection and their 2D position coordinates and thus allows us to extract three-dimensional (3D) momentum. Appropriate tuning gates select only those electron signals synchronized with the single bunches and the TOF spectra of the electrons are measured with respect to the bunch marker of the synchrotron radiation source. We record only events in which at least two ions and one electron are detected in coincidence. The orientation of the molecular axis at the time of photoemission is extracted from the momentum vectors of the $\mathrm{CO}^{+}$and $\mathrm{O}^{+}$fragments resulting from Coulomb dissociation of $\mathrm{CO}_{2}^{2+}$ subsequent to rapid Auger decay. The electron energy and angular resolution varies as a function of energies. The energy resolutions are $0.5 \mathrm{eV}$ and $4 \mathrm{eV}$ at photon energies of $543 \mathrm{eV}$ and $574 \mathrm{eV}$, respectively, while the angular resolutions are $12^{\circ}$ and $5^{\circ}$ at $543 \mathrm{eV}$ and $574 \mathrm{eV}$, respectively. The ion energy and angle resolutions are $0.5 \mathrm{eV}$ and $2^{\circ}$, respectively; they are constant for different photon energies.

Our calculated MF-PADs were obtained within a partially relaxed-core Hartree-Fock (partially RCHF) approximation with a molecular basis set obtained using Slater's transitionstate approximation [29]. In this approximation the relaxed molecular orbitals are derived from a self-consistent calculation where half an electron is removed from the oxygen $\mathrm{K}$ shell. This procedure attempts to capture effects arising from screening of the K-shell hole while maintaining the calculation at the one-electron level. To avoid working with non-orthogonal orbitals, we also use this molecular basis to construct the initial N-electron state and the final N-electron state with an electron in the continuum [10]. To obtain the photoelectron orbitals we used an iterative procedure to solve the Lippmann-Schwinger equation associated with the one-electron Schrödinger equation [30] with a potential produced by the transition-state orbitals. In figure 1, the calculated $\mathrm{O} 1 \mathrm{~s}$ photoionization spectrum is compared with the measured total-ion-yield (TIY) spectrum, which is equivalent to the absorption spectrum. The maxima at the photon energies of $\sim 543$ and $560 \mathrm{eV}$ are well reproduced by the calculations and are attributed to the $5 \sigma_{g}^{*}$ and $4 \sigma_{u}^{*}$ resonances, respectively, as previously assigned [15]. The TIY spectrum includes the contributions from the valence photoionization. Even if this is taken into account, the ratio of the resonant to the direct $\mathrm{O}$ $1 \mathrm{~s}$ photoionization cross sections is overestimated by the present calculation. The measured spectrum exhibits another maximum at $\sim 544 \mathrm{eV}$ between the two shape resonances, whereas the calculated one does not. The maximum at $\sim 544 \mathrm{eV}$ in the measured spectrum is attributed to the double excitations [15], which are not included in the calculation. The measured $4 \sigma_{u}^{*}$ resonance is broader than the calculated one, though the apparent broadening may partly come from the appearance due to the baseline of TIY which is much higher than the calculated ones and partly come from the double excitations neglected in the calculations.

Figure 2 shows our measured (dots) and calculated (lines) MF-PADs for $\mathrm{O} 1 \mathrm{~s}$ photoemission from $\mathrm{CO}_{2}$, at photon energies of 543, 547, 550, 554, 559, 564 and $574 \mathrm{eV}$. Our apparatus collects all ions and electrons ejected in $4 \pi$ directions. In the data analysis, however, we select the events in which the electron is ejected in the plane spanned by the electric vector and the molecular orientation. To do this, in the laboratory frame, we define 18 planes, which include the electric vector, by $10^{\circ}$ step. If the dipole approximation is fulfilled, then the angular correlations in the events in all these planes are equivalent. In fact, we could not see any anomaly in the events in any of these planes within the experimental statistics. Thus we summed them over. In this way, the experimental MF-PADs in figure 2 are obtained. The molecular axis for both the experimental and theoretical data is horizontal and the electric

9 See http://roentdek.com for details on the detectors. 


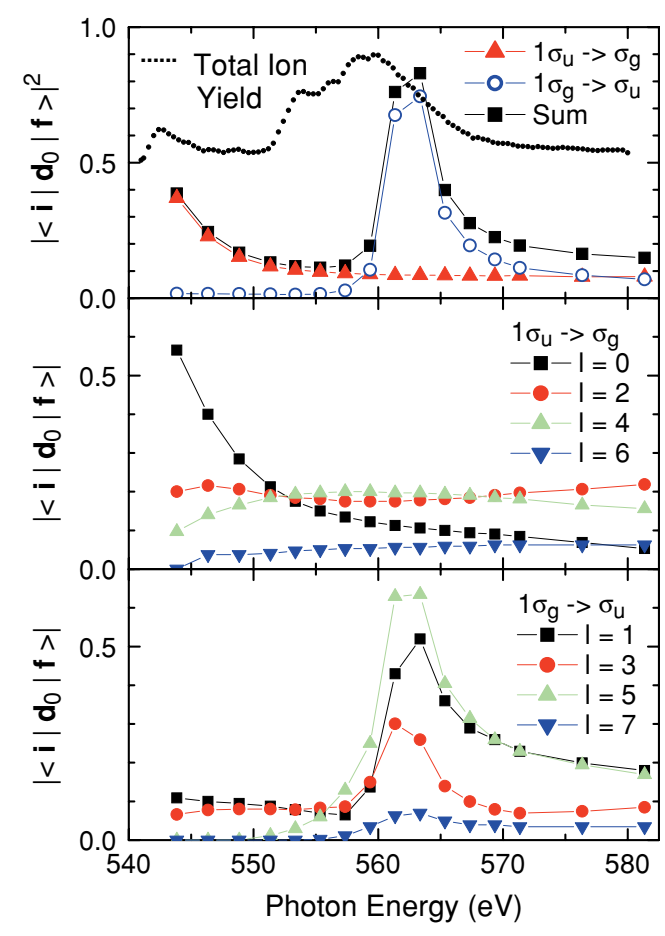

Figure 1. Total-ion-yield spectrum (dots) of $\mathrm{CO}_{2}$ in the energy region of the $\mathrm{O} 1 \mathrm{~s}$ ionization region, in an arbitrary scale, recorded with a photon energy resolution of $120 \mathrm{meV}$, and the amplitudes of the dipole moments for the partial waves for the $1 \sigma_{u} \rightarrow \sigma_{g}$ and $1 \sigma_{g} \rightarrow \sigma_{u}$ channels calculated within a partially RCHF approximation.

vector of the light is at $0 \pm 7.5^{\circ}, 45 \pm 7.5^{\circ}$ and $90 \pm 7.5^{\circ}$ with respect to the molecular axis. The distributions with the electric vector at $0^{\circ}$ and $90^{\circ}$ correspond to $\Sigma \rightarrow \Sigma$ (referred to as $\Sigma$ ) and $\Sigma \rightarrow \Pi(\Pi)$ transitions, respectively. The angle resolutions in the MF-PADs are estimated to be $\sim 15^{\circ}$, which come from both the experimental angular resolutions and the angular acceptance (a bin size of $5^{\circ}$ ) in making the plots. For better comparison with the measured data, the calculated MF-PADs in figure 2 have been convoluted with a Gaussian distribution with a FWHM of $15^{\circ}$. The distribution at each energy is normalized so that the integrated intensity (area enclosed) for the $\Pi$ channel is unity for both the measured and calculated spectra. The distributions for different orientations of the molecular axis $\left(0^{\circ}, 45^{\circ}\right.$ and $90^{\circ}$ ) are on relative scales.

We first focus on the MF-PADs for the $\Sigma$ channel, where the electric vector is parallel to the molecular axis. The partial wave composition $(l=0,2,4$ and 6$)$ of the calculated photoionization amplitudes can provide useful insight into the angular distributions in this channel. Figure 1 also includes these amplitudes. In this channel, there are two contributions, $1 \sigma_{g} \rightarrow \sigma_{u}$ and $1 \sigma_{u} \rightarrow \sigma_{g}$. Even partial wave components arise in photoionization of the $1 \sigma_{u}$ orbital. In the vicinity of the $5 \sigma_{g}^{*}$, located close to the threshold, we find that a $l=0$ component is dominant. As the photon energy increases, the amplitude of the $l=0$ component decreases rapidly and higher $l(2$ and 4$)$ contributions become dominant. In the photoionization of the $1 \sigma_{g}$ orbital, on the other hand, odd partial wave components arise. In addition to a substantial p $(l=1)$ component expected for ionization of a $1 \sigma_{g}(\mathrm{O} 1 \mathrm{~s})$ orbital, higher $l$ ( 3 and 5) contributions are seen to be significant, not only at the shape resonance around 


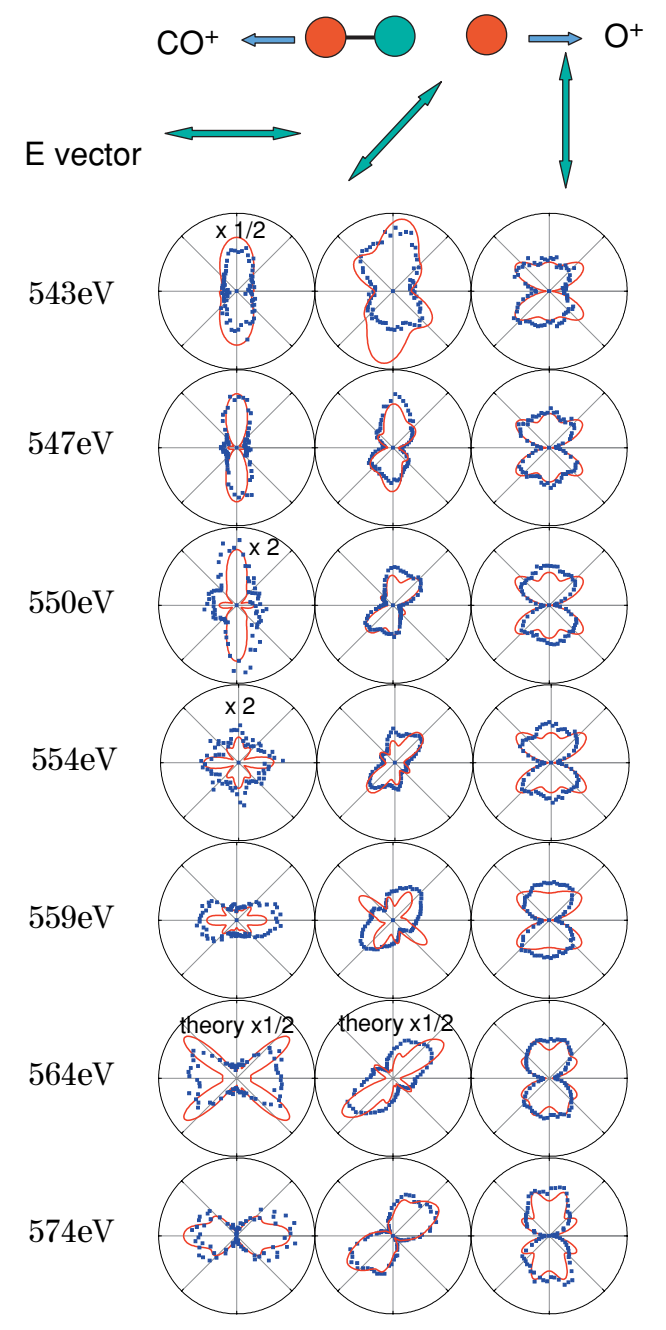

Figure 2. MF-PADs for $\mathrm{O} 1 \mathrm{~s}$ photoemission from $\mathrm{CO}_{2}$ molecules at photon energies of 543, 547, 550, 554, 559, 564 and $574 \mathrm{eV}$. The dots and curves correspond to measurements and calculations, respectively. The direction of electric vector, $E$, is horizontal. The molecular axis is at $0 \pm 7.5^{\circ}, 45 \pm 7.5^{\circ}$ and $90 \pm 7.5^{\circ}$ with respect to $E$.

a photon energy of $\sim 560 \mathrm{eV}$, but also at lower and higher photoelectron energies. The calculated angular distributions in the $\Sigma$ channel in figure 2 reproduce the general features of the measured spectra reasonably well, except at the photon energy of $\sim 564 \mathrm{eV}$. Agreement between the calculated and measured MF-PADs at the photon energy of $564 \mathrm{eV}$ in the $\Sigma$ channel is clearly less satisfactory than at the lower energies in figure 2. The calculated distribution displays sharp structure which is not apparent in the measured spectra. A similar disagreement in the shape resonance region was seen in the previous study on MF-PADs for C 1s photoemission from $\mathrm{CO}_{2}$ [12]. Finally, the calculated and measured MF-PAD at the highest photoelectron energy studied here is quite good. A careful inspection of the measured MFPADs reveals that the MF-PADs display a reflection asymmetry for the plane perpendicular to the molecular axis. The $\mathrm{O} 1 \mathrm{~s}$ photoelectrons are preferentially ejected in the direction of 


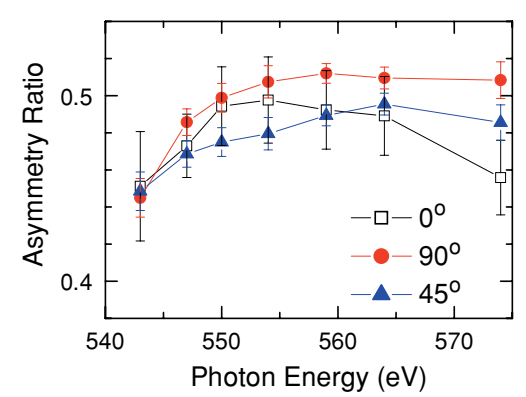

Figure 3. Ratios of yields to the one side to the total as a function of the photon energy and orientation of the electric vector relative to the molecular axis. See the text about the side.

fragmentation of the $\mathrm{O}^{+}$ions for almost all photon energies. This asymmetry feature will be discussed later.

In the $\Pi$ channel, where the electric vector is perpendicular to the molecular axis and no shape resonance is seen, the shapes of the calculated and measured angular distributions are in good agreement. The measured MF-PADs display asymmetry for the plane perpendicular to the molecular axis. The $\mathrm{O} 1 \mathrm{~s}$ photoelectrons are preferentially ejected in the direction of fragmentation of the $\mathrm{CO}^{+}$ions near the $\mathrm{O} 1 \mathrm{~s}$ threshold. This tendency is opposite to the case of the $\Sigma$ channel.

Figure 2 also shows measured and calculated MF-PADs for an orientation of $45^{\circ}$ between the electric vector and the molecular axis. These distributions are determined by a coherent mixture of the $\Sigma$ and $\Pi$ channels and both the ratios of amplitudes and phases in these channels play a significant role. These distributions can hence be additional benchmarks for testing theoretical methods and models. In general, agreement between the calculated and measured distributions is reasonable, except for the photon energy of $559 \mathrm{eV}$, and suggests that the calculated phase relationship between the $\Sigma$ and $\Pi$ channels is satisfactory. The calculated MF-PADs show point symmetry whereas the measured MF-PADs show asymmetry near the $\mathrm{O} 1 \mathrm{~s}$ threshold. The $\mathrm{O} 1 \mathrm{~s}$ photoelectrons are preferentially ejected in the direction of the larger of the two sectors between the fragmentation direction of $\mathrm{CO}^{+}$ions and the $E$ vector.

These MF-PADs for $\mathrm{O} 1 \mathrm{~s}$ photoemission from $\mathrm{CO}_{2}$ display weak but non-negligible reflection asymmetry for the plane perpendicular to the molecular axis for $0^{\circ}$ and $90^{\circ}$, and reflection asymmetry for the centre of mass of the molecule for $45^{\circ}$. The result may be rationalized as follows. Anti-symmetric stretching vibrations are known to arise in the $\mathrm{O} 1 \mathrm{~s}$ ionized state $[17,18]$. Coupled with this vibrational motion, the $\mathrm{CO}_{2}^{+}$ion has an asymmetric equilibrium configuration for the plane perpendicular to the molecular axis. Thus one can expect that the $\mathrm{C}-\mathrm{O}$ bond, with the oxygen atom containing the core hole, elongates and results in $\mathrm{O}^{+}-\mathrm{CO}^{+}$fragmentation. In this case, the core hole resides on the $\mathrm{O}^{+}$ion and an ejected electron thus experiences an asymmetric potential resulting in a reflection asymmetry in the MF-PADs. We note, however, that the asymmetry has been diluted. If the detection of the $\mathrm{O}^{+}$ were a 1:1 correspondence of the localized core hole, we would expect very strong reflection asymmetry in the MF-PADs but the observed asymmetry is rather weak.

To see these asymmetries quantitatively, we estimate the ratio for yields of the one side relative to the total yields for each MF-PAD and plot it in figure 3 as a function of photon energy for the three orientations of the electric vector relative to the molecular axis. In the figure the ratios for $0^{\circ}$ and $90^{\circ}$ are given by the counts of the left and right sides, respectively, divided by the total counts, whereas the ratio for $45^{\circ}$ is given by the counts of the upper 
side with respect to the line perpendicular to the electric vector, divided by the total counts. The asymmetry is stronger at the lowest energies and becomes weaker with the increase in energy. The asymmetry for $0^{\circ}$ increases again at higher energy. We note that the momentum $k$ of the photoelectron at photon energy $574 \mathrm{eV}$ is $2.93 \AA^{-1}$ while the $\mathrm{O}-\mathrm{O}$ distance $R$ is $2.324 \AA$ and thus $k R \simeq 2 \pi$. Therefore the observed oscillation of the asymmetry for $0^{\circ}$ may stem from the interference effect corresponding to the extended $x$-ray absorption fine structure.

In conclusion, we have reported on the results of measurements and calculations of MFPADs for $\mathrm{O} 1 \mathrm{~s}$ photoemission from $\mathrm{CO}_{2}$, not only for the $\Sigma$ and $\Pi$ channels, where the molecular axis is parallel and perpendicular to the electric vector, but also for the molecular axis oriented at $45^{\circ}$ to the electric vector. The agreement between the measured and calculated MF-PADs is on average very reasonable. The MF-PADs display a weak reflection asymmetry through the plane perpendicular to the molecular axis which is attributed to symmetry lowering due to the anti-symmetric stretching vibrations.

\section{Acknowledgments}

The measurements were carried out with approval of the SPring-8 Program Review Committee and supported in part by grants-in-aid for scientific research from the Japan Society for the Promotion of Science. The authors are grateful to the staff of SPring- 8 for their help in the course of these studies.

\section{References}

[1] Shigemasa E, Adachi J, Oura M and Yagishita A 1995 Phys. Rev. Lett. 74359

[2] Heiser F, Ge $\beta$ ner G, Viefhaus J, Wieliczek K, Hentges R and Becker U 1997 Phys. Rev. Lett. 792435

[3] Landers A et al 2001 Phys. Rev. Lett. 87013002

[4] Cherepkov N A, Semenov S K, Hikosaka Y, Ito K, Motoki S and Yagishita A 2000 Phys. Rev. Lett. 84250

[5] Motoki S, Adachi J, Hikosaka Y, Ito K, Sano M, Soejima K, Yagishita A, Raseev G and Cherepkov N A 2000 J. Phys. B: At. Mol. Opt. Phys. 334193

[6] Weber Thet al 2001 J. Phys. B: At. Mol. Opt. Phys. 343669

[7] Jahnke T et al 2002 Phys. Rev. Lett. 88073002

[8] Adachi J, Hosaka K, Furuya S, Soejima K, Takahashi M, Yagishita A, Semenov S K and Cherepkov N A 2003 Phys. Rev. Lett. 91163001

[9] Jahnke T et al 2004 Phys. Rev. Lett. 93083002

[10] Cherepkov N A, Raseev G, Adachi J, Hikosaka Y, Ito K, Motoki S, Sano M, Soejima K and Yagishita A 2000 J. Phys. B: At. Mol. Opt. Phys. 334213

[11] Adachi J, Motoki S, Cherepkov N A and Yagishita A 2002 J. Phys. B: At. Mol. Opt. Phys. 355023

[12] Saito N et al 2003 J. Phys. B: At. Mol. Opt. Phys. 36 L25

[13] Golovin A V, Adachi J, Motoki S, Takahashi M and Yagishita A 2005 J. Phys. B: At. Mol. Opt. Phys. 38 L63

[14] Prince K C, Avaldi L, Coreno M, Camilloni R and de Simone M 1999 J. Phys. B: At. Mol. Opt. Phys. 322551

[15] Wight G R and Brion C E 1974 J. Electron Spectrosc. Relat. Phenom. 3191

[16] Watanabe N, Adachi J, Soejima K, Shigemasa E and Yagishita A 1997 Phys. Rev. Lett. 784910

[17] Domcke W and Cederbaum L S 1977 Chem. Phys. 25189

[18] Kivimäki A, Kempgens B, Maier K Köppe H M, Piancastelli M N, Neeb M and Bradshaw A M 1997 Phys. Rev. Lett. 79998

[19] Tanaka T and Kitamura H 1996 J. Synchrotron Radiat. 347

[20] Ohashi H, Ishiguro E, Tamenori Y, Kishimoto H, Tanaka M, Irie M, Tanaka T and Ishikawa T 2001 Nucl. Instrum. Methods A 467-468 529

[21] Ohashi H et al 2001 Nucl. Instrum. Methods A 467-468 533

[22] Yoshida H, Senba Y, Morita M, Goya T, De Fanis A, Saito N, Ueda K, Tamenori Y and Ohashi H 2004 AIP Conf. Proc. 705267

[23] De Fanis A et al 2002 Phys. Rev. Lett. 89023006 
[24] Saito N, Ueda K and Koyano I 2003 AIP Conf. Proc. 652172

[25] De Fanis A et al 2004 J. Phys. B: At. Mol. Opt. Phys. 37 L235

[26] Hanold K A, Garner M C and Continetti R E 1996 Phys. Rev. Lett. 773335

[27] Lafosse A, Lebech M, Brenot J C, Guyon P M, Jagutzki O, Spielberger L, Vervloet M, Houver J C and Dowek D 2000 Phys. Rev. Lett. 845987

[28] Moshammer R et al 1994 Phys. Rev. Lett. 733371

[29] Slater J C 1974 The Self-Consistent Field for Molecules and Solids: Quantum Theory of Molecules and Solids vol 4 (New York: McGraw-Hill)

[30] Lucchese R R, Raseev G and McKoy V 1982 Phys. Rev. A 252572 